\title{
Comparison of the closed-loop identification methods in terms of the bias distribution
}

\author{
Alireza Karimi* , Ioan Doré Landau \\ Laboratoire d'Automatique de Grenoble (CNRS-INPG-UJF), ENSIEG, BP. 46, 38402 Saint Martin d'Hères, France
}

Received 17 April 1997; accepted 11 November 1997

\begin{abstract}
Several schemes for plant model identification in closed-loop operation including classical direct method, two-step identification and closed-loop output error algorithms are considered. These methods are analyzed and compared in terms of the bias distribution of the estimates for the case that the noise model is estimated as well as the case that a fixed model of noise is considered (output error structure). The problems concerning the filtered direct method which is often used in the iterative identification and control scheme are mentioned. It is shown that these problems may be solved by the closed-loop output error identification method. (c) 1998 Elsevier Science B.V. All rights reserved.
\end{abstract}

Keywords: Closed-loop identification; Bias distribution; Identification for control; Output error

\section{Introduction}

Identification of plant models from closed-loop data is an important practical issue and may be motivated by the following reasons:

(1) There are plants which contain an integrator or are unstable in open-loop operation.

(2) The performance of the closed-loop system can be improved using a controller based on the identified model from the closed-loop data. This can be theoretically proved when the true system belongs to the model set and for three different control design criteria (minimum variance, LQG and model reference control) $[3,4]$. The measure of the performance is the variance of the error between the output of the optimal closed-loop system (with the optimal controller computed from

\footnotetext{
* Corresponding author. Tel.: +33 4768264 81; fax: +33 476 8263 88; e-mail: karimi@lag.ensieg.inpg.fr.
}

the true system) and that of the actual closedloop system (with the controller computed from the identified model).

(3) The research on identification for robust control shows that a plant model identified in closed-loop operation is more precise in the critical frequency zone (cross-over frequency) for the robust control design. This idea led to the well-known iterative identification and control scheme. In this scheme the identification is performed with the closed-loop data filtered by an appropriate filter which depends on the objective of the control. It can be shown that when the true system is not in the model set using these methods one tends to minimize an upper bound on an achieved cost [10]. The achieved cost is, in fact, the norm of error between the achieved closedloop performance (the true system with the designed controller) and the designed closedloop performance (the identified model and the designed controller). 
In view of the importance of the closed-loop identification, several schemes have been developed and analyzed in the literature. Two surveys of these methods can be found in $[2,14]$.

The asymptotic expressions of bias distribution of transfer function estimates play an important role in comparing different methods, particularly, when the true system does not belong to the model set. This analysis is often done for the case that a fixed model of noise is considered $[9,13]$. In this paper we present the bias distribution of the estimates for different schemes while the noise model is also estimated. This will clarify the effect of filtering and of the estimated noise model on the bias distribution.

One of the problems in closed-loop identification is that there is a correlation between output noise and plant input. This problem has been generally neglected using the hypothesis that either the closed-loop signals are noise free [1] or the spectrum of excitation signal that enters the loop dominates the noise spectrum within the closed-loop bandwidth [2, 17]. A solution to this problem using the dual Youla parameterization is given in [11] and for a particular case is given by the so-called two-stage identification in [13]. Recently, a family of closed-loop output error algorithms has been also developed which gives unbiased estimation of the plant model parameters in the presence of noise [5-7]. The unbiasedness of these algorithms for the case that the true system belongs to the model set has been proved in the mentioned papers and here the distribution of bias will be given when the true system is not in the model set. It will be shown subsequently that the implicit filtering effect included in these algorithms makes them a useful tool for control relevant identification.

The contribution of this paper is to give the bias distribution of the estimates for a recently developed family of recursive algorithms [5-7] and compare them with the classical methods. The bias distribution of the closed-loop identification methods while the noise model is not fixed will be also presented. This allows one to establish that some methods are more suitable than others for control relevant identification.

The paper is organized as follows: In Section 2 as an introduction, the bias distribution of the classical direct method for closed-loop identification is mentioned. Then the bias distribution for the two-stage indirect identification method and the filtered direct methods for the case that the noise model is estimated are presented in Sections 3 and 4, respectively. The bias distributions for the closed-loop output error iden- tification algorithms are given in Section 5. A simulation example will be presented in Section 6 and some concluding remarks will be given in Section 7 .

\section{Direct method}

In this method the input/output data of the plant operating in closed loop are used to identify the plant model [12], so it is not necessary that the controller be known. The classical open-loop identification algorithms may be used to identify the plant model.

Consider a discrete time SISO system with additive disturbances

$y(t)=G\left(q^{-1}\right) u(t)+H\left(q^{-1}\right) e(t)$,

where $y(t)$ is the output signal, $u(t)$ is the input signal and $e(t)$ is a zero-mean unit variance white noise signal. $G\left(q^{-1}\right)$ and $H\left(q^{-1}\right)$ are the transfer function operators and $q^{-1}$ is the delay operator.

An optimal predictor, in the sense that the prediction error tends asymptotically to a white noise, for the output is given by [8]

$$
\begin{aligned}
\hat{y}(t)= & \hat{H}^{-1}\left(q^{-1}, \rho\right)\left[\hat{G}\left(q^{-1}, \theta\right) u(t)\right. \\
& \left.+\left(\hat{H}\left(q^{-1}, \rho\right)-1\right) y(t)\right],
\end{aligned}
$$

where $\hat{y}(t)$ is a one-step ahead predictor of the output and $\hat{G}\left(q^{-1}, \theta\right)$ and $\hat{H}\left(q^{-1}, \rho\right)$ are the estimates of $G\left(q^{-1}\right)$ and $H\left(q^{-1}\right)$, respectively. The parameter vectors $\theta$ and $\rho$ describe the plant and noise model, respectively. For the ease of notation $q^{-1}, \rho$ and $\theta$ may be dropped out in the remainder of the paper.

The prediction error $\varepsilon(t)$ can be computed as follows:

$$
\varepsilon(t)=\hat{H}^{-1}[(G-\hat{G}) u(t)+(H-\hat{H}) e(t)]+e(t) .
$$

It is clear that if $\hat{G} \rightarrow G$ and $\hat{H} \rightarrow H$ then the prediction error tends to a white noise.

For an infinite number of samples the parameter estimates are obtained by the minimization of the expectation of the squared prediction error

$\theta^{*}=\arg \min _{\theta, \rho} E\left\{\varepsilon^{2}(t)\right\}$.

The second term in the right-hand side of Eq. (2.3) does not play any role in the minimization of Eq. (2.4) and can be ignored. On the other hand, for the case 


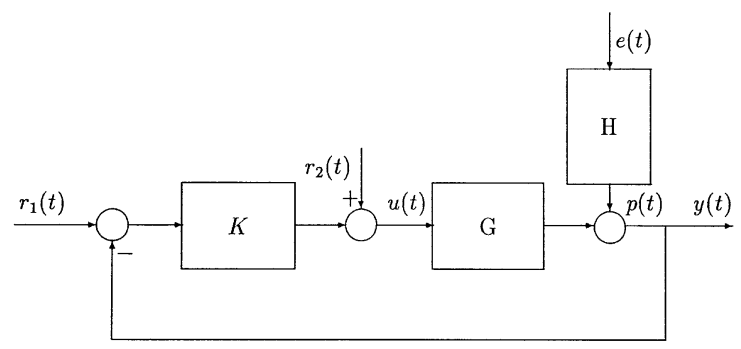

Fig. 1. Closed-loop system.

of open-loop identification $e(t)$ and $u(t)$ are independent, so using the Parseval's relation Eq. (2.4) can be represented by an integral in the frequency domain

$$
\begin{aligned}
\theta^{*}= & \arg \min _{\theta, \rho} \int_{-\pi}^{\pi}\left|\hat{H}^{-1}\left(\mathrm{e}^{\mathrm{j} \omega}\right)\right|^{2}\left[\mid G\left(\mathrm{e}^{\mathrm{j} \omega}\right)\right. \\
& -\left.\hat{G}\left(\mathrm{e}^{\mathrm{j} \omega}\right)\right|^{2} \phi_{u}(\omega) \\
& \left.+\left|H\left(\mathrm{e}^{\mathrm{j} \omega}\right)-\hat{H}\left(\mathrm{e}^{\mathrm{j} \omega}\right)\right|^{2} \phi_{e}(\omega)\right] \mathrm{d} \omega,
\end{aligned}
$$

where $\phi_{u}(\omega)$ and $\phi_{e}(\omega)$ are the spectrum of the input $u(t)$ and the noise $e(t)$. In the sequel $\mathrm{e}^{\mathrm{j} \omega}$ is also omitted whenever there is no risk of confusion.

In the case of closed-loop identification, denoting by $K\left(q^{-1}\right)$ the controller operating during identification, the plant input can be expressed as (Fig. 1)

$u(t)=T_{u r} r(t)+S_{u p} H e(t)$,

where $T_{u r}$ is the transfer function between the excitation signal and the plant input and $S_{u p}$ is the input sensitivity function defined by

$S_{u p}=\frac{-K}{1+G K}$.

$T_{u r}$ is equal to the output sensitivity function $S_{y p}$ when the excitation signal is added to the plant input $\left(r_{2}(t)=r(t), r_{1}(t)=0\right)$ or equal to the input sensitivity function with a negative sign $\left(T_{u r}=-S_{u p}\right)$ while the excitation signal is added to the control input $\left(r_{1}(t)=r(t), r_{2}(t)=0\right)$. The output sensitivity function is defined as

$S_{y p}=\frac{1}{1+G K}$.

Substituting $u(t)$ from Eq. (2.6) into Eq. (2.3) one obtains

$$
\begin{aligned}
\varepsilon(t)= & \hat{H}^{-1}\left\{(G-\hat{G}) T_{u r} r(t)\right. \\
& \left.+\left[(G-\hat{G}) S_{u p} H+(H-\hat{H})\right] e(t)\right\}+e(t) .
\end{aligned}
$$

It can be observed from the above equation that even in closed-loop operation the predictor of Eq. (2.2) is an optimal predictor.

By applying the Parseval's relation one gets the bias distribution of the estimates in closed-loop operation

$$
\begin{aligned}
\theta^{*}= & \arg \min _{\theta, \rho} \int_{-\pi}^{\pi}\left|\hat{H}^{-1}\right|^{2}\left[|G-\hat{G}|^{2}\left|T_{u r}\right|^{2} \phi_{r}(\omega)\right. \\
& \left.+\left|(G-\hat{G}) S_{u p} H+(H-\hat{H})\right|^{2} \phi_{e}(\omega)\right] \mathrm{d} \omega .
\end{aligned}
$$

Comparing this equation with Eq. (2.5), one observes that in the closed-loop operation the bias of the plant model is related to the bias of the noise model. It means that contrary to the open-loop case even when the noise model and plant model are independently parameterized, incorrect estimation of the noise model leads to the bias estimation of the plant model. It can be shown that this bias is proportional to the noise variance and $H-\hat{H}$ [9]. This bias effect becomes more evident when the noise model is not estimated (output error structure) or a fixed model of noise is considered. In this case in the predictor of Eq. (2.2) $\hat{H}$ is replaced by 1 and the following one-step ahead output error predictor is obtained

$\hat{y}(t)=\hat{G} u(t)$.

Using this predictor, the prediction error is computed as follows:

$\varepsilon(t)=(G-\hat{G}) T_{u r} r(t)+\left[(G-\hat{G}) S_{u p}+1\right] H e(t)$.

Using again the Parseval's relation, one obtains

$$
\begin{aligned}
\theta^{*}= & \arg \min _{\theta} \int_{-\pi}^{\pi}\left[|G-\hat{G}|^{2}\left|T_{u r}\right|^{2} \phi_{r}(\omega)\right. \\
& \left.+\left|(G-\hat{G}) S_{u p}+1\right|^{2}|H|^{2} \phi_{e}(\omega)\right] \mathrm{d} \omega .
\end{aligned}
$$

It is evident that the minimization of the two terms in the above integral leads to a biased estimation of the plant model and for large noise variance $\hat{G}$ approaches $-1 / K$. However, since the standard tools for openloop identification can be utilized for minimizing this criterion, this method is often used when the spectrum of the excitation signal dominates the spectrum of the noise signal. If it is not the case the indirect twostage method or the special closed-loop identification algorithms should be used. 


\section{Two-stage identification}

In this method identification of the plant model operating in closed loop is performed in two stages [13]. In the first stage, the transfer function between the excitation signal and the plant input is identified in an open-loop way. Then the excitation signal filtered by the identified transfer function is used as an instrumental variable to generate a noiseless input for identifying the plant model in the second stage.

Suppose that the transfer function $T_{u r}^{*}$ is identified in the first stage and the noiseless instrumental variable for the plant input is

$u^{*}(t)=T_{u r}^{*} r(t)$.

Then the one-step ahead predictor for the output of the system is

$\hat{y}(t)=\hat{H}^{-1}\left[\hat{G} u^{*}(t)+(\hat{H}-1) y(t)\right]$.

Now, the prediction error is given by

$\varepsilon(t)=\hat{H}^{-1}\left[G u(t)-\hat{G} u^{*}(t)+(H-\hat{H}) e(t)\right]+e(t)$.

Using Eqs. (2.6) and (3.1) one gets

$$
\begin{aligned}
\varepsilon(t)= & \hat{H}^{-1}\left\{\left(G T_{u r}-\hat{G} T_{u r}^{*}\right) r(t)\right. \\
& \left.+\left[\left(G S_{u p}+1\right) H-\hat{H}\right] e(t)\right\}+e(t) .
\end{aligned}
$$

Replacing now $G S_{u p}+1$ by $S_{y p}$, one obtains

$$
\begin{aligned}
\varepsilon(t)= & \hat{H}^{-1}\left[\left(G T_{u r}-\hat{G} T_{u r}^{*}\right) r(t)\right. \\
& \left.+\left(S_{y p} H-\hat{H}\right) e(t)\right]+e(t) .
\end{aligned}
$$

The term $e(t)$ is independent of the rest and can be left out of the calculation. Then the parameter estimates is approximated by

$$
\begin{aligned}
\theta^{*}= & \arg \min _{\theta, \rho} \int_{-\pi}^{\pi}\left|\hat{H}^{-1}\right|^{2}\left[\left|G T_{u r}-\hat{G} T_{u r}^{*}\right|^{2} \phi_{r}(\omega)\right. \\
& \left.+\left|S_{y p} H-\hat{H}\right|^{2} \phi_{e}(\omega)\right] \mathrm{d} \omega .
\end{aligned}
$$

From these results one can conclude that

- Estimation of the noise model is biased, in fact, the estimated noise model $\hat{H}$ tends to $S_{y p} H$ which is the real noise model acting on the closed-loop output.

- In the case that the plant and noise model are independently parameterized, the biasedness of the noise model does not influence the estimation of the plant model.
- The quality of the estimation of the plant model depends upon the estimation of the $T_{u r}$ in the first stage, so a poor estimation of $T_{u r}$ gives a biased estimation of the plant model. This situation as suggested in [13] may be avoided by choosing a high order transfer function (or FIR) for estimation of $T_{u r}$ in the first stage.

- In the ideal case i.e. $\hat{H}=S_{y p} H$ and $T_{u r}^{*}=T_{u r}\left(=S_{y p}\right.$ when the excitation signal is added to the plant input) we have

$\theta^{*}=\arg \min _{\theta} \int_{-\pi}^{\pi}\left|H^{-1}\right|^{2}\left[|G-\hat{G}|^{2} \phi_{r}(\omega)\right] \mathrm{d} \omega$,

which shows that the bias distribution is affected by the spectrum of the excitation signal instead of the spectrum of the plant input. Thus, this method gives the same bias distribution as open-loop identification methods and the benefits of identification in closed-loop operation may not be obtained by this method.

In the case that the output error structure is considered for identification in the second stage the noise model is not estimated and we have $\hat{H}=1$ which leads to the following prediction error:

$$
\varepsilon(t)=\left(G T_{u r}-\hat{G} T_{u r}^{*}\right) r(t)+S_{y p} H e(t) .
$$

Then the bias distribution is given by

$$
\begin{aligned}
\theta^{*}= & \arg \min _{\theta} \int_{-\pi}^{\pi}\left[\left|G T_{u r}-\hat{G} T_{u r}^{*}\right|^{2} \phi_{r}(\omega)\right. \\
& \left.+\left|S_{y p} H\right|^{2} \phi_{e}(\omega)\right] \mathrm{d} \omega .
\end{aligned}
$$

It is observed that using an output error structure in the ideal case $\left(T_{u r}^{*}=T_{u r}\right)$ the bias distribution is only influenced by the spectrum of the plant input.

\section{Filtered direct methods}

Fig. 2 illustrates the scheme which is often used in the context of iterative combination of identification in closed loop and control re-design. The upper parts represents the true closed-loop system (the designed controller and the real plant) while the lower part represents the design system (the designed controller and the identified plant model). The problem of joint identification and control then can be formulated as follows:

$$
\min _{\theta} J(G, K(\hat{G}(\theta))) .
$$




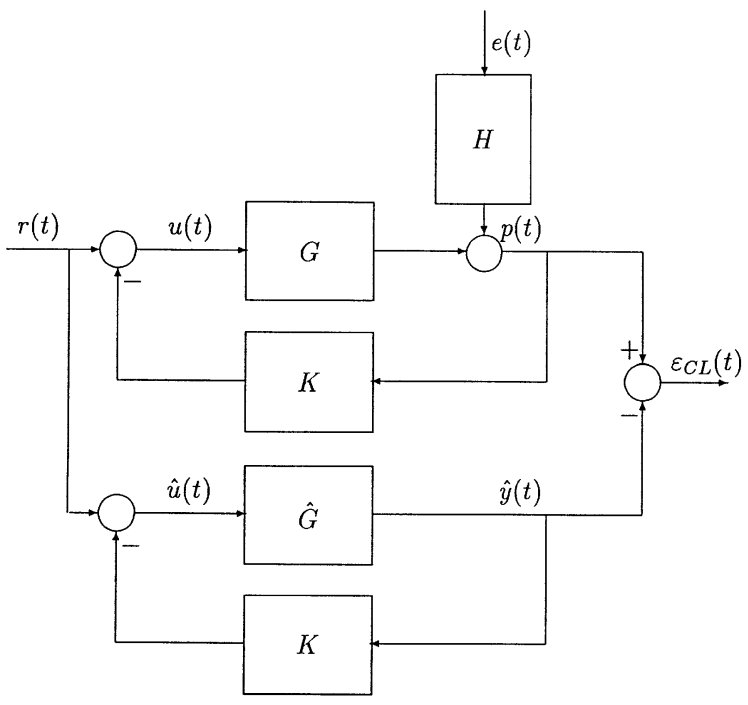

Fig. 2. The scheme of identification for control.

Since the direct minimization of the above criterion is not typically tractable, a succession of local identification steps and local control design steps can be carried out iteratively. The key point in the local identification steps is that the identification criterion should be made to match a criterion which is the difference between the achieved and the designed costs. This idea was proposed for LQG control in [16], for $H_{\infty}$ control in [10] and for pole placement in [1]. According to these results in order to minimize a robust stability criterion in the local identification steps the infinity norm of the following expression should be minimized:

$(G-\hat{G}) \hat{S}_{u p}$

where $\hat{S}_{u p}=-K(1+\hat{G} K)^{-1}$ is the input sensitivity function in the designed closed-loop system. Since no feasible algorithm is presently available for the $H_{\infty}$ identification, a least-squares method is usually used instead. In the same way, for minimizing a robust performance criterion related to a tracking objective the following criterion should be minimized in the local identification steps [2, 14]:

$\hat{S}_{y p}(G-\hat{G}) T_{u r}$,

where $\hat{S}_{y p}=(1+\hat{G} K)^{-1}$ is the output sensitivity function in the designed closed-loop system.

Generally, this criterion can be minimized using an appropriate time-varying data filter for the data acquired in the closed-loop operation and using a standard identification method. This data filter normally depends upon the parameters of the model which is identified and cannot be directly used by the standard identification methods. An approximation of this filter is used based on the parameters of the most recent parameter estimates available. Then a direct identification is performed on the filtered data in order to obtain the parameters of the plant model. For this case (filtered direct method) the filtering of input/output data leads to the following prediction error (the prediction error of Eq. (2.9) is filtered by $\left.L\left(q^{-1}\right)\right)$ :

$$
\begin{aligned}
\varepsilon(t)= & \hat{H}^{-1} L\left\{(G-\hat{G}) T_{u r} r(t)+\left[(G-\hat{G}) S_{u p} H\right.\right. \\
& \left.\left.+\left(H-\frac{\hat{H}}{L}\right)\right] e(t)\right\}+e(t) .
\end{aligned}
$$

Consequently, the bias distribution is obtained as follows:

$$
\begin{aligned}
\theta^{*}= & \arg \min _{\theta, \rho} \int_{-\pi}^{\pi}\left|\hat{H}^{-1} L\right|^{2}\left[|G-\hat{G}|^{2}\left|T_{u r}\right|^{2} \phi_{r}(\omega)\right. \\
& \left.+\left|(G-\hat{G}) S_{u p} H+\left(H-\frac{\hat{H}}{L}\right)\right|^{2} \phi_{e}(\omega)\right] \mathrm{d} \omega .
\end{aligned}
$$

The following comments can be given for this criterion:

- The estimation of the noise model is biased. In fact, $\hat{H}$ tends asymptotically to $L H$ instead of $H$.

- A poor estimation of $L H$ by $\hat{H}$ occurs when either the plant and noise model are not independently parameterized (using ARX and ARMAX models) or the order of $\hat{H}$ is not sufficiently large. This poor estimation leads to the bias estimation of $G$ even when $G$ is in the model set.

- In the ideal case when $\hat{H}=L H$ the parameter estimates is given by

$$
\begin{aligned}
\theta^{*}= & \arg \min _{\theta} \int_{-\pi}^{\pi}\left|H^{-1}\right|^{2}\left[| G - \hat { G } | ^ { 2 } \left(\left|T_{u r}\right|^{2} \phi_{r}(\omega)\right.\right. \\
& \left.\left.+\left|S_{u p} H\right|^{2} \phi_{e}(\omega)\right)\right] \mathrm{d} \omega
\end{aligned}
$$

which means that the data filtering by $L$ will be compensated by the noise model $\hat{H}$ and the bias distribution is almost identical to the non-filtered case.

- We can choose $L=S / P_{0}$ where $S$ is the denominator of the controller and $P_{0}$ is an estimation of the 
closed-loop characteristic polynomial (this special filter is proposed in [1] for matching the identification criterion with the pole placement control criterion). Now, if an ARX model is considered (i.e. $\hat{H}=1 / \hat{A}$ where $\hat{A}$ is the estimation of the denominator of the plant model $\hat{G}$ ) for the identified model we have:

$$
\begin{aligned}
\theta^{*}= & \arg \min _{\theta} \int_{-\pi}^{\pi}\left|S_{y p}^{*}\right|^{2}\left[|G-\hat{G}|^{2}\left|T_{u r}\right|^{2} \phi_{r}(\omega)\right. \\
& \left.+\left|(G-\hat{G}) S_{u p} H+\left(H-\left(S_{y p}^{*}\right)^{-1}\right)\right|^{2} \phi_{e}(\omega)\right] \mathrm{d} \omega,
\end{aligned}
$$

where $S_{y p}^{*}=\hat{A} S / P_{0}$ is an estimation of the output sensitivity function $S_{y p}$. It can be observed that the estimation of $G-\hat{G}$ is frequency weighted by multiplication of two sensitivity functions which resembles to the robust performance criterion of Eq. (4.3) but the effect of noise is still present.

It is suggested in [15] that if an output structure is used for closed-loop identification the data should be filtered by an estimation of the output sensitivity function $S_{y p}^{*}$. For this case the prediction error of Eq. (2.12) is filtered by $S_{y p}^{*}$ and gives the following bias distribution:

$$
\begin{aligned}
\theta^{*}= & \arg \min _{\theta} \int_{-\pi}^{\pi}\left|S_{y p}^{*}\right|^{2}\left[|G-\hat{G}|^{2}\left|T_{u r}\right|^{2} \phi_{r}(\omega)\right. \\
& \left.+\left|(G-\hat{G}) S_{u p}+1\right|^{2}|H|^{2} \phi_{e}(\omega)\right] \mathrm{d} \omega .
\end{aligned}
$$

Evidently, the estimation of the plant model using the above criterion like the direct case remains biased. The bias distribution of $G-\hat{G}$ is similar to the general case using an ARX model.

Remarks. The major problems of the filtered direct methods can be noted as follows:

1. The parameter estimates are asymptotically biased in the presence of noise even when the true system belongs to the model set.

2. The data filters depend on the model that is being identified and are not fixed.

3. In the case that the noise model is also estimated, the filtering effect is compensated by the estimated noise model.

The source of these problems is that one tries to use the standard open-loop identification methods for an identification problem which has a totally different structure. A solution is to develop a special identification method for the desired structure (i.e. the structure presented in Fig. 2).

\section{Closed-loop output error methods}

A family of recursive algorithms, so-called CLOE, can be used [5-7] in order to identify a plant model which minimizes directly the closed-loop prediction error ( $\varepsilon_{C L}$ in Fig. 2$)$ between the achieved closedloop system and the designed one. These algorithms are based on a re-parameterized adjustable predictor for the closed-loop system in terms of a known fixed controller and an adjustable plant model.

Consider the system described by Eq. (2.1) and the following output error predictor for the closed-loop system:

$\hat{y}(t)=\hat{G} \hat{u}(t)$,

where $\hat{u}(t)=r(t)-K \hat{y}(t)$ when the excitation signal is added to the plant input. For the case that the excitation signal is added to the control input we have $\hat{u}(t)=K(r(t)-\hat{y}(t))$. However, these two cases lead finally to the same prediction error.

The prediction error is given by

$\varepsilon(t)=G u(t)+H e(t)-\hat{G} \hat{u}(t)$.

Adding and subtracting $G \hat{u}(t)$ to the right-hand side of the above equation one gets:

$\varepsilon(t)=(G-\hat{G}) \hat{u}(t)+G(u(t)-\hat{u}(t))+H e(t)$.

Replacing $u(t)$ and $\hat{u}(t)$ by the control law, one obtains:

$\varepsilon(t)=(G-\hat{G}) \hat{u}(t)-G K(y(t)-\hat{y}(t))+H e(t)$,

which leads to

$\varepsilon(t)=S_{y p}[(G-\hat{G}) \hat{u}(t)+H e(t)]$.

Substituting now $\hat{u}(t)$ by $\hat{T}_{u r} r(t)$ where $\hat{T}_{u r}$ is the transfer function between $r(t)$ and $\hat{u}(t)$ the bias distribution will be obtained as follows:

$$
\begin{aligned}
\theta^{*}= & \arg \min _{\theta} \int_{-\pi}^{\pi}\left|S_{y p}\right|^{2}\left[|G-\hat{G}|^{2}\left|\hat{T}_{u r}\right|^{2} \phi_{r}(\omega)\right. \\
& \left.+|H|^{2} \phi_{e}(\omega)\right] \mathrm{d} \omega .
\end{aligned}
$$

This criterion shows that:

- The estimation of the plant model parameters is unbiased when $G$ is in the model set. 
- The bias distribution is not affected by the spectrum of the noise (which is the case for the filtered direct method).

- The bias distribution is affected by the multiplication of two sensitivity functions which is exactly the desired frequency weighting of Eq. (4.3) (it can easily be shown that $\hat{S}_{y p} T_{u r}=S_{y p} \hat{T}_{u r}$ ).

- An approximation of this criterion (when the excitation signal is added to the plant input i.e. $T_{u r}=S_{y p}$ ) may be obtained by the two-step identification method if:

1. the estimation of $T_{u r}$ in the first stage is not biased,

2. the data (the noiseless input $u^{*}(t)$ and the plant output $y(t))$ filtered by $T_{u r}^{*}$ are used for the identification in the second stage,

3. an output error structure is considered in the second stage.

Two other algorithms of this family named F-CLOE and AF-CLOE [6] have exactly the same bias distribution. Because the differences are at the level of the observation vector used in the parameter adaptation algorithm and for all three algorithms (CLOE, F-CLOE and AF-CLOE) the prediction error has exactly the same structure.

There are also two extensions of these algorithms in which the noise model is also identified (X-CLOE and G-CLOE [7]). For these algorithms the optimal predictor for the closed-loop system is

$\hat{y}(t)=\hat{G} \hat{u}(t)+\hat{\bar{H}} \varepsilon(t)$,

where

$\hat{\bar{H}}=\hat{H}-\hat{S}_{y p}^{-1}$.

Remark. For an ARMAX model we have $\hat{H}=\hat{C} / \hat{A}$ and $\hat{G}=\hat{B} / \hat{A}$ then the predictor will be (with $K=R / S$ )

$\hat{y}(t)=\hat{G} \hat{u}(t)+\frac{\hat{C} S-\hat{A} S-\hat{B} R}{\hat{A} S} \varepsilon(t)$,

which is the predictor used in X-CLOE method.

The prediction error then can be computed as follows:

$\varepsilon(t)=G u(t)+H e(t)-\hat{G} \hat{u}(t)-\hat{\bar{H}} \varepsilon(t)$.

Similar to the previous case the prediction error can be written as

$$
\varepsilon(t)=(G-\hat{G}) \hat{u}(t)-G K \varepsilon(t)-\hat{\bar{H}} \varepsilon(t)+H e(t) .
$$

Because of the form of the predictor in this case, $\hat{u}(t)$ is affected by the noise via $\varepsilon(t)$ in the predictor so one has

$\hat{u}(t)=\hat{T}_{u r} r(t)-K \hat{\bar{H}} \hat{S}_{y p} \varepsilon(t)$.

Replacing $\hat{u}(t)$ from the above equation in Eq. (5.9) one gets

$$
\begin{aligned}
(1 & \left.+G K+(G-\hat{G}) K \hat{\bar{H}} \hat{S}_{y p}+\hat{\bar{H}}\right) \varepsilon(t) \\
& =(G-\hat{G}) \hat{T}_{u r} r(t)+H e(t)
\end{aligned}
$$

and one obtains

$$
\begin{aligned}
\varepsilon(t)= & S_{y p}\left(1+\hat{\bar{H}} \hat{S}_{y p}\right)^{-1}\left[(G-\hat{G}) \hat{T}_{u r} r(t)+H e(t)\right] \\
= & \left(1+\hat{\bar{H}} \hat{S}_{y p}\right)^{-1}\left[S_{y p}(G-\hat{G}) \hat{T}_{u r} r(t)\right. \\
& \left.+H S_{y p} e(t)-\left(1+\hat{\bar{H}} \hat{S}_{y p}\right) e(t)\right]+e(t),
\end{aligned}
$$

which leads to the following bias distribution (denoting $\left.\bar{H}=H-S_{y p}^{-1}\right)$ :

$$
\begin{aligned}
\theta^{*}= & \arg \min _{\theta, \rho} \int_{-\pi}^{\pi}\left|\left(1+\hat{\bar{H}} \hat{S}_{y p}\right)^{-1}\right|^{2} \\
& \times\left[\left|S_{y p}\right|^{2}|G-\hat{G}|^{2}\left|\hat{T}_{u r}\right|^{2} \phi_{r}(\omega)\right. \\
& \left.+\left|\bar{H} S_{y p}-\hat{\bar{H}} \hat{S}_{y p}\right|^{2} \phi_{e}(\omega)\right] \mathrm{d} \omega .
\end{aligned}
$$

This bias distribution can also be reformulated as

$$
\begin{aligned}
\theta^{*}= & \arg \min _{\theta, \rho} \int_{-\pi}^{\pi}\left|\left(1+\hat{\bar{H}} \hat{S}_{y p}\right)^{-1} S_{y p}\right|^{2} \\
& \times\left[|G-\hat{G}|^{2}\left|\hat{T}_{u r}\right|^{2} \phi_{r}(\omega)\right. \\
& \left.+\left|(G-\hat{G}) \hat{S}_{u p} \hat{\bar{H}}+(\bar{H}-\hat{\bar{H}})\right|^{2} \phi_{e}(\omega)\right] \mathrm{d} \omega .
\end{aligned}
$$

It can be observed that:

- This method gives an unbiased estimation of the plant and noise model when they are in the model set.

- It has the same asymptotic properties as the direct identification method in terms of the bias distribution. In fact, if in Eq. (5.13) we replace $\bar{H}$ and $\hat{\bar{H}}$, respectively, by $H-S_{y p}^{-1}$ and $\hat{H}-\hat{S}_{y p}^{-1}$, Eq. (2.10) is obtained.

- If the noise model is not estimated (i.e. $\hat{\bar{H}}=0$ ) the CLOE method can be derived from this method.

- Like the filtered direct method in the X-CLOE and G-CLOE the estimation of the noise model will 

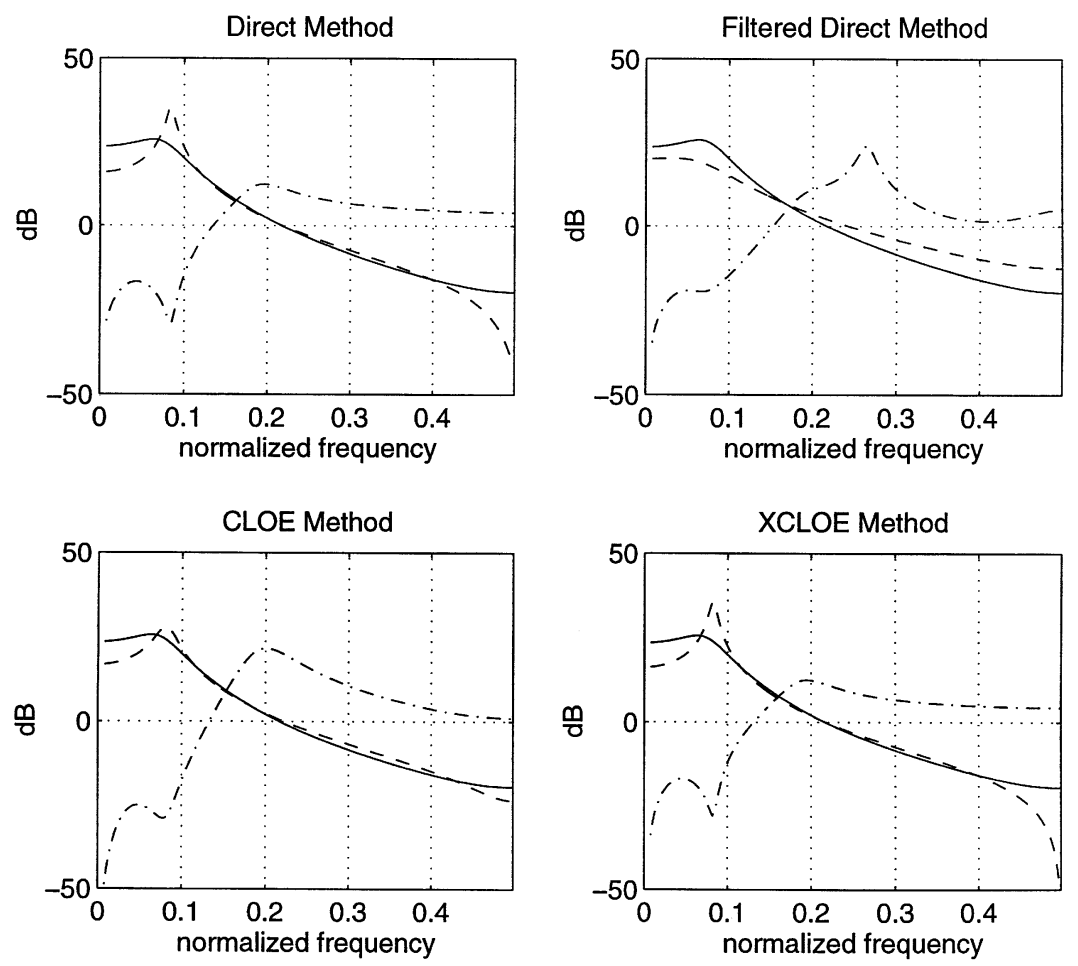

Fig. 3. Magnitude of the frequency response of: (-) real system; (- -) identified plant model; (--) the filter acting on the excitation spectrum.

compensate the inherent filtering effect of the CLOE method (replace $\left(1+\hat{\bar{H}} \hat{S}_{y p}\right)$ by $\hat{H} \hat{S}_{y p}$ in Eq. (5.13)).

\section{Simulation example}

In this section a simulation example is presented in order to demonstrate the effects of noise on the bias distribution of different closed-loop identification methods. In this simulation a discrete-time third-order system operating in closed loop using a fixed and known controller in the feedback will be identified with a second-order model in the presence of noise. The real system is given by

$$
G\left(q^{-1}\right)=\frac{q^{-1}\left(1+0.5 q^{-1}\right)}{\left(1-1.5 q^{-1}+0.7 q^{-2}\right)\left(1-0.5 q^{-1}\right)}
$$

and the controller by

$$
K\left(q^{-1}\right)=\frac{0.8659-1.2763 q^{-1}+0.5204 q^{-2}}{\left(1-q^{-1}\right)\left(1+0.3717 q^{-1}\right)} .
$$

A uniformly distributed zero-mean white noise is added to the output of the system. The variance of noise is about $35 \%$ of the output variance. The closeloop system is excited with a PRBS generated by a 8-bit shift register and length of 512 sampling period.

Fig. 3 shows the magnitude of the frequency response of the identified models comparing with that of the real system using the direct method, filtered direct method, closed-loop output error (CLOE) and extended closed-loop output error method. A secondorder model of the system is identified in open-loop operation and is used to compute the input/output data filter for the filtered direct method. For the direct and filtered direct method an ARMAX model is considered. The frequency response of the filter acting on the spectrum of the excitation signal is also plotted in order to show the effect of this filter on the bias distribution. It can be observed that:

- the minimum bias is obtained in the frequency zone where the magnitude of the frequency response of the filter acting on the input spectrum is maximum,

- the bias distribution for the CLOE method is not affected by noise while for the other methods the bias is larger in the zone where the magnitude of the frequency response of the filter is low, 
- the XCLOE method and direct method have the same bias distribution.

\section{Conclusions}

Several schemes for plant model identification in closed-loop operation have been compared in terms of the bias distribution of the estimates. The results show that for open-loop-type methods used for "control relevant identification" there is no hope that the bias distribution approaches the desired one in the presence of noise. It has also been shown that a recently developed family of algorithms for plant model identification in closed loop gives on one hand a bias distribution which is not influenced by noise and, on the other hand, contains an implicit frequency weighting filter which is matched with a robust performance control criterion. These properties make this algorithm a very suitable tool for control relevant identification.

\section{Editorial note}

This paper discusses issues and results which are similar to the investigations in the paper by U. Forssell and L. Ljung, "Issues in Closed Loop Identification", paper LiTH-ISY-R-1940, 1997. This paper has been published on the understanding that both research group in Linköping and Grenoble have developed these ideas independently.

\section{References}

[1] K.J. Åström, Matching criteria for control and identification, European Control Conf., Groningen, Netherlands, 1993, pp. 248-251.

[2] M. Gevers, Towards a joint design of identification and control? in: H.L. Trentelman, J.C. Willems (Eds.), Essays on Control, Perspectives in the Theory and its Applications, Birkhäuser, Boston, USA, 1993.

[3] M. Gevers, L. Ljung, Optimal experiment designs with respect to the intended model application, Automatica 22(5) (1986) 543-554.

[4] H. Hjalmarsson, M. Gevers, F. De Bruyne, J. Leblond, Identification for control: closing the loop gives more accurate controllers, in: Conf. on Decision and Control, Lake Buena Vista, FL, USA, 1994, pp. 4150-4155.

[5] I.D. Landau, A. Karimi, An extended output error recursive algorithm for identification in closed loop, in: 35th Conf. on Decision and Control, Kobe, Japan, 1996.

[6] I.D. Landau, A. Karimi, An output error recursive algorithm for unbiased identification in closed loop, Automatica 33(5) (1997) 933-938.

[7] I.D. Landau, A. Karimi, Recursive algorithms for identification in closed loop - a unified approach and evaluation, Automatica 33(8) (1997) 1499-1523.

[8] L. Ljung, System Identification - Theory for the User, Prentice-Hall, Englewood Cliffs, NJ, 1987.

[9] L. Ljung, Information contents in identification data from closed-loop operation, in: Conf. on Decision and Control, San Antonio, Texas, 1993, pp. 2248-2252.

[10] R.R. Schrama, Accurate identification for control: the necessity of an iterative scheme, IEEE Trans. Automat. Control 37(7) (1992) 991-994.

[11] R.R. Schrama, P.M.J. Van den Hof, An iterative scheme for identification and control design based on coprime factorizations, in: American Control Conf., Chicago, IL, USA, 1992, pp. 2842-2846.

[12] T. Söderström, P. Stoica, System Identification, Prentice-Hall, Englewood Cliffs, NJ, 1989.

[13] P.M.J. Van den Hof, R.R. Schrama, An indirect method for transfer function estimation from closed loop data, Automatica 29 (1993) 1523-1527.

[14] P.M.J. Van den Hof, R.R. Schrama, Identification and control - closed-loop issues, Automatica 31(12) (1995) 1751-1770.

[15] A. Voda, I.D. Landau, An iterative method for the autocalibration of the digital controllers application, in: European Control Conf., Rome, Italy, 1995, pp. 2463-2468.

[16] Z. Zang, R.R. Bitmead, M. Gevers, Iterative model refinement and control robustness enhancement, in: Conf. on Decision and Control, Brighton, UK, 1991, pp. 279-284.

[17] Z. Zang, R.R. Bitmead, M. Gevers, Iterative weighted leastsquares identification and weighted LQG control design, Automatica 31(11) (1995) 1577-1594. 